\title{
A New ECT Image Reconstruction Algorithm Based on Convolutional Neural Network
}

\author{
Lanying $\mathrm{Li}^{*}$, Yin Kong and Jianda Sun \\ The College of Computer Science and Technology, Harbin University of Science \\ and Technology, Harbin 150080, China \\ lulu08521@sina.com
}

\begin{abstract}
In order to solve the problem of image reconstruction in electrical capacitance tomography (ECT) technology, the feasibility of applying convolutional neural network $(C N N)$ to ECT image reconstruction is studied. The convolution layer and the training of the structure of the sub sampling method is improved based on deep research for convolution neural network for the more time-consuming process of deep structure and training issues, and a fast convergence convolution neural network (FCCNN) image reconstruction method is proposed. Matlab was used to build a ECT simulation system. For each algorithm, the simulation results were compared and analyzed. The experimental results show that our algorithm improved the image reconstruction efficiency and quality of the common flow pattern.
\end{abstract}

Keywords: capacitance tomography; convolution neural network; image reconstruction; sub sampling; Matlab

\section{Introduction}

In 1991, Leeds University and Schlumberger research companies in England completed the world's first real-time electrical capacitance tomography (ECT) system engineering and successfully applied in petroleum industry of multiphase flow measurement [1]. Since then, ECT technology has been one of the hot spots in multiphase flow process tomography [2]. But the application of ECT is still limited by the measurement precision of the system and the quality of image reconstruction and the speed of the system in the process of solving [3]. So many researchers have pay more attention to the design optimization of the sensor and the image reconstruction algorithm [4].

ECT image reconstruction algorithm can be divided into three categories: direct algorithm, iterative algorithm and new algorithm [5]. The direct algorithm has fast imaging speed and simple structure, but the linearization process has lost a lot of important information, so the results of imaging quality is not high, and the distortion is large. This kind of algorithms are mainly linear back projection (LBP) method, singular value decomposition and the Tikhonov regularization method, and so on. The iterative algorithm is characterized by its high image quality compared with the non-iterative one, but the imaging speed of iterative algorithm was slow for its relative complex structure. Mainly Newton-Raphsom algorithm, Tikhonov iterative algorithm and Landweber algorithm are typical iterative algorithms and are widely used nowadays [6-7], So improving the efficiency of image reconstruction algorithm and maintaining the high quality in the same time has become the key point of current research.

In recent years, researchers have developed new type algorithms, including image reconstruction methods such as the application of neural networks genetic algorithm,

${ }^{*}$ Corresponding Author 
linear Kalmam filter algorithm, support vector machines and deep learning algorithms, etc. The research and application of this kind of algorithm can effectively improve the quality and speed of image reconstruction, which provides a new direction for the research of ECT system [8].

Traditional neural networks are shallow layer of learning networks, so they have insufficient ability for network extracts features and expression with less hidden layers [9]. These features lead to the final imaging accuracy not high. But neural networks has high fault tolerance and highly nonlinear neural network description ability, which can realize the complex nonlinear mapping between intelligent optimization measurement data and flow pattern, and can solve the "soft field" problem of ECT system effectively. So this kind of intelligent algorithm was intensively studied by researchers and was applied to explore the electrical capacitance tomography inversion problem [10-11]. On the contrary, a major feature of the deep neural network is containing more hidden layer, which can express the image feature information well. But deep neural network is difficult to train and time consuming. The convolutional neural network is a kind of typical deep learning model. In 1998, the deep convolutional neural network was applied to the recognition of handwritten numerals by Lecu, and obtained good results. Benefit from the weight sharing characteristic of the convolutional neural network, the gradient diffusion of the convolution layer can effectively avoided [12]. We found that the deep structure characteristics of the convolution neural network has a great improvement on the lack of feature extraction and the low accuracy of the imaging precision by analyzing the algorithm. In addition, the improved algorithm can solve the speed of the iterative algorithm to compensate the slow convergence of the iterative algorithm by the characteristics of the local receiving region and the weight sharing.

In this paper, In order to improve the imaging speed and quality of the reconstructed image, the existing ECT image reconstruction algorithms and the system characteristics are researched, and the improved depth network CNN algorithm is applied in image reconstruction of ECT.

\section{ECT Introduction}

ECT system mainly consists of three modules: the sensor module, data acquisition module and the computer image reconstruction module [13] (Figure 1). The principle is based on different dielectric constants in the pipeline to be measured. Firstly, get the capacitor information of any two plates through the sensor. Secondly, the data signal is processed and amplified by the data acquisition module, and then transmitted to the computer for image reconstruction.

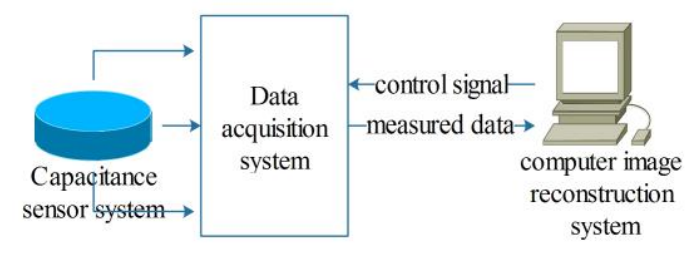

Figure 1. Structure of ECT System

\subsection{ECT Imaging Principle}

There are two mathematical problems in ECT Technology: forward and inverse problems [14]. The forward problem is how to solve the potential distribution, charge quantity and capacitance value of each plate in the condition of the known boundary condition, the dielectric constant and the medium distribution. The main ideas in the study of positive problems can be expressed as follows: 
$C^{E \times 1}=S^{E \times N} \cdot G^{N \times 1}$

Where $C \in R_{E}, \quad \mathrm{C}$ is $E \times 1$ dimensional normalized projection capacitance vector. $S \in R_{E \times N}$ is the sensitivity coefficient matrix. $G \in R_{N}$ is the normalized distribution medium gray image vector. $N$ represents the number of measured capacitance values for each frame image. The number of measured independent capacitor for $\mathrm{n}$ electrode ECT system is: $C_{n}^{2}=n \cdot(n+1) / 2$. Capacitance between any two plates can be expressed as:

$C_{i j}=\iint_{D} \varepsilon(x, y) S_{i j}(x, y,(x, y)) d x d y$

Where $C_{i j}$ represents the capacitance values of electrode between $\mathrm{i}$ and $\mathrm{j}$. $D$ indicates the imaging area of measured pipeline. $\varepsilon(x, y)$ is the distribution function of dielectric in the pipe. $S_{i j}(x, y)$ represents the sensitivity distribution of capacitance value at the pipe cross section point $(x, y)$ between $\mathrm{i}$ and $\mathrm{j}$ plate. The model continuous space is infinite model. So the data in sensitive field can't be directly operated. We should translate the double integral operation into the differential unit area summation based on their physical significance, and solve the capacitance values and the normalized sensitivity matrix. The Finite Element Method (FEM) [15-16] is used in this paper.

Anti-question refers to the problem based on the data measured and calculated in the forward problem, which is the image reconstruction problem of ECT from Formula (1). The inverse problem model can also be expressed as:

$G^{N \times 1}=S^{N \times E} C^{E \times 1}$

Formula (3) mainly described the nonlinear continuous model relationships between image and capacitance values. In order to improve the system's image quality and precision, the researchers hope to get more system measurement data by increasing the number of plates, from 6 to 12 or 16 electrodes. However, the more plates increase the measurement difficult of weak capacitance value and the interference factors, and the cost also increases. Image reconstruction algorithm is one important aspect of imaging. Since the capacitor number in 12 electrode system which can be measured is 66 , the number is far less than the pixel subdivision of the pipeline. As the sensitivity coefficient inverse matrix does not exist in Formula (1), the Formula (3) is underdetermined equation, leading to solutions of this equation not unique. In order to express more accurately the non-linear relationship of Formula (1) and (3), we need to choose a good image reconstruction algorithm. A new convolutional neural network algorithm is used in this paper. The key point of this algorithm is to use $\mathrm{CNN}$ to describe the mapping of the nonlinear relationship between the capacitance value and the medium distribution, and to obtain the output image media distribution normalized gray value by inputting the normalized capacitance values.

\section{Improved Convolutional Neural Network Algorithm}

Convolutional neural network (CNN) is a kind of depth neural network with supervised learning, and the unique structure of local sensing and shared weights makes CNN have more advantage in the field of image recognition and processing. Local perception makes the image's local spatial pixels more closely, and the shared weight reduces the number of network training parameters and the calculation complexity. But its complex network structure and the time-consuming training process are insufficient for a higher real-time requirements ECT systems. As to the characteristics and requirements of $\mathrm{CNN}$ and ECT 
imaging system, the CNN was improved and verified, and a fast convergence convolution neural network (called FCCNN) image reconstruction method is proposed. The overall structure of the CNN is shown in Figure 2.

FCCNN is similar to the traditional CNN structure in the network structure. But the convolution implementation process of FCCNN is different from the traditional algorithms. The traditional convolution layer contains a large number of convolution kernels, which makes the convolution layer structure very complex. Since the convolution process takes up most of the time of this algorithm, for sub-sampling layer the entire CNN computing time accounted for less than $1 \%$ [17-18]. So the speed convolution layer can effectively improve the efficiency of the algorithm. This paper will change the traditional convolution model into four sub convolution modes. The algorithm implementation process is that a top feature map only generates four mapping feature map after convolution algorithm. The convolution process can enhance the original signal feature and reduce the noise effectively. Its discrete convolution process is as follows:

$$
X_{j}^{L+1}=f\left(\sum_{i \in M_{j}} X_{i}^{L} \cdot K_{i j}^{L+1}+b_{j}^{L+1}\right)
$$

The $L+1$ layer denotes convolution layer, it is connected with the upper layer through the convolution operation, and is connected to the next layer by down-sampling operation. $K$ represents a convolution kernel, $b$ represents an offset term. Where $f(\cdot)$ is the activation function of the network. This paper selects the sigmoid function as $f(x)=1 /\left(1+e^{-x}\right),[-\infty,+\infty]$ is mapped to $[0,1]$.

Detailed convolution process is shown in Figure 2.

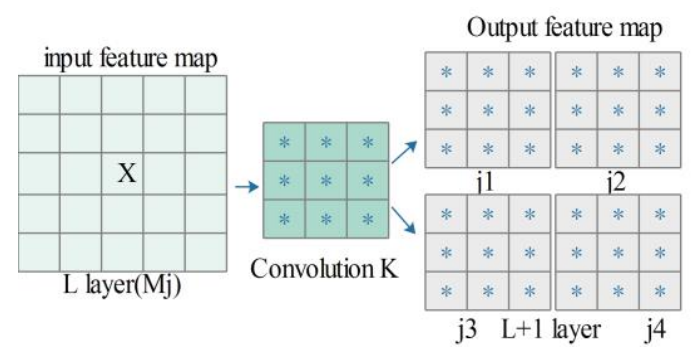

Figure 2. Improved Convolution Layer

As to the insufficient problem of extracted feature maps which is resulted in by four samples, for each pool sample area $\mathrm{R}$, in the sampling process, the real time complexity and variability are taken into account for the various flow patterns, and the algorithm also changes the pool sampling function. Due to the traditional maximum sampling function too curing and single, it may excessively enlarge the activation values in training set, and did not give opportunities to those small implicit activation values for spreading information to the top, that reduces the generalization capability of the network. The mean sampling value is the calculation result of the all activation values. So there may be no way for mean value to represent the majority of the activation values of the sampling pool area. Therefore, the paper uses a random square pool sampling method instead of traditional Maximum sampling and Mean sampling method. Different from the traditional sampling method, the random square pool sampling method took into account the possibility of the emergence of each element. Due to the algorithm characteristics of randomly selecting network data information, from the view of the whole network, more information can be transmitted to the next layer of the structure. At the same time, it also can prevent over fitting phenomenon due to the small amount of data. The specific approach is to calculate the square of activation value as a basis for the sampling probability. Firstly, calculate the square of the activation value $y_{i}$ of each element in the 
pool sampling area $R$, and then get the emergence probability o the element $p_{i}$. Its implementation process is as follows:

$$
P_{i}=\frac{y_{i}^{2}}{\sum_{k \in R} y_{k}^{2}} \text {, and } \sum_{i \in R} P_{\mathrm{i}}=1
$$

After getting the discrete probability distribution set $P_{i}(i \in R)$, the sampling value $Q$ is obtained by random sampling according to the probability distribution set $P_{i}$, the pool sampling value $H$ is defined as:

$$
H=Q \text { and } Q \sim P_{i}\left(p_{1}, p_{2} \ldots p_{R}\right)
$$

Specific examples of the calculation process is shown in Figure 3:

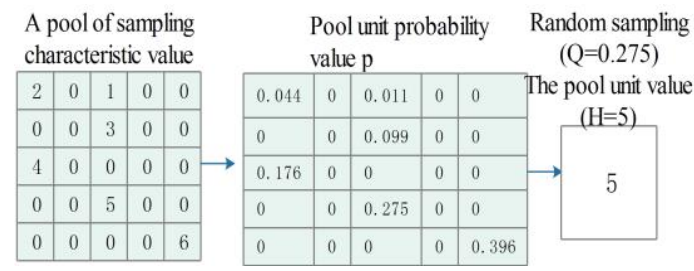

Figure 3. Process of Pool Sampling

The FCCNN network structure is divided into seven layers, which includes an input layer (the capacitance value is normalized), an output layer (the 1432 image gray value), two convolution layer (the convolution kernel size is $2 * 2$ ), 2 sampling layer and a full connection layer. The training of FCCNN is similar to that of the traditional BP algorithm, which is mainly divided into three steps:

Firstly, the basic parameters of the network weights are set, including the setting of learning of convolution kernel, connection weights, threshold and bias parameters etc.

Secondly, in the process of network forward propagation, a batch of normalized capacitance value training data is entered, and the network takes the supervised learning method. The actual output vector $T_{p}$ is obtained by the forward calculation step by step, and is delivered to the output layer. The actual output is calculated as Equation (7).

$T_{p}=F_{n}\left(\ldots\left(F_{2}\left(F_{1}\left(X_{P} W^{(1)}\right) W^{(2)}\right) \ldots\right) W^{n}\right)$

Thirdly, the back-propagation procedure: calculating the minimum variance between the actual output $T_{p}$ and the corresponding ideal output vector $Y_{p}$, and according to the minimization error method of back-propagation adjust each weight and bias. The method is as follows:

$w(t+1)=w(t)+\eta \delta(t) x(t)$

In the Formula (8), $w(t)$ is the weight, $x(t)$ is the output of the neuron, $\delta(t)$ expresses the error term of the neuron, and $\eta$ indicates the learning rate. So repeat, until the set value of error indicators or the maximum number of training steps. For the problem of a multi class classification, including $N$ training and $C$ categories, the individual sample data error in all the training is an error of the whole data set, the error function formula for sample data sets are as follows: 
$E_{p}=\frac{1}{2} \sum_{n=1}^{N} \sum_{k=1}^{C}\left(y_{k}^{n}-t_{k}^{n}\right)^{2}$

Where $y_{k}^{n}$ denotes the ideal output value for the input $k$ data in sample $n, t_{k}^{n}$ represents the actual network output value after the $k$ data in sample $n$ is calculated by the feature extraction.

\section{Simulation and Analysis of ECT Image Reconstruction}

In simulation system, we can use the finite element method to triangulate the test region into three layers: pipe, pipe wall and pipe shield. The pixel number of three layer is 1452, 1248 and 768. The specific parameters of capacitance sensor are set as shown in Table 1. ECT sensor finite element triangulation is shown in Figure 4.

Table 1. ECT Sensor Module Parameter Setting

\begin{tabular}{|c|c|c|}
\hline Pipe radius & plates & plate angle \\
\hline $\begin{array}{c}66 \mathrm{~mm} \\
\text { Radial electrode } \\
\text { depth } \\
6 \mathrm{~mm}\end{array}$ & $\begin{array}{c}12 \\
\text { pipe wall } \\
\text { thickness } \\
12 \mathrm{~mm}\end{array}$ & $\begin{array}{l}\text { 22degrees } \\
\text { shield } \\
\text { thickness } \\
10 \mathrm{~mm}\end{array}$ \\
\hline $\begin{array}{c}0.1 \\
0.08 \\
0.06 \\
0.04 \\
0.02 \\
0.02 \\
0.04 \\
-0.02 \\
-0.04 \\
-0.08 \\
-0.04 \\
-0.1\end{array}$ & $\begin{array}{ll}0 & 1 \\
\text { unit }: m & 0.05\end{array}$ & \\
\hline
\end{tabular}

Figure 4. Triangulation Results of Finite Element

The simulation software is Matlab which runs on a computer: Intel core I3 2.4GHZ CPU, 2G memory and win7 operating system. The test data were collected in three typical two-phase flow pattern: laminar flow, droplet flow and annular flow. Three prototypes flow patterns are shown in Figure 5.

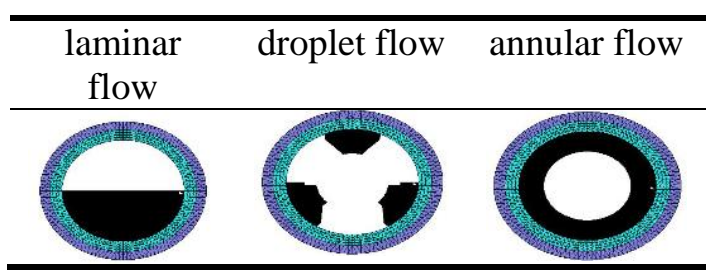

Figure 5. Prototype Flow

All the experimental data were obtained from the simulation system. There are a total of 2000 sets of training data for each flow pattern samples. A total of 6000 sample data set is used for pre-training, testing and validation of CNN and FCCNN. The oil / water is used as experimental medium inside the tubes, and the relative dielectric constant of oil and water is 2.5 and 80 . The output threshold is 41.25 , and Pixel will is output as high 
medium when the value of Pixel is higher than 41.25, low medium otherwise. In order to make the image clear and reduce the influence of other factors and image artifacts, the reconstructed image is represented by two values, the high medium is represented by the black image region, and the low medium is represented by the white image area. The above three kinds of flow patterns are reconstructed by LBP algorithm, Landweber algorithm, CNN and improved FCCNN algorithm. The results are shown in Figure 6.

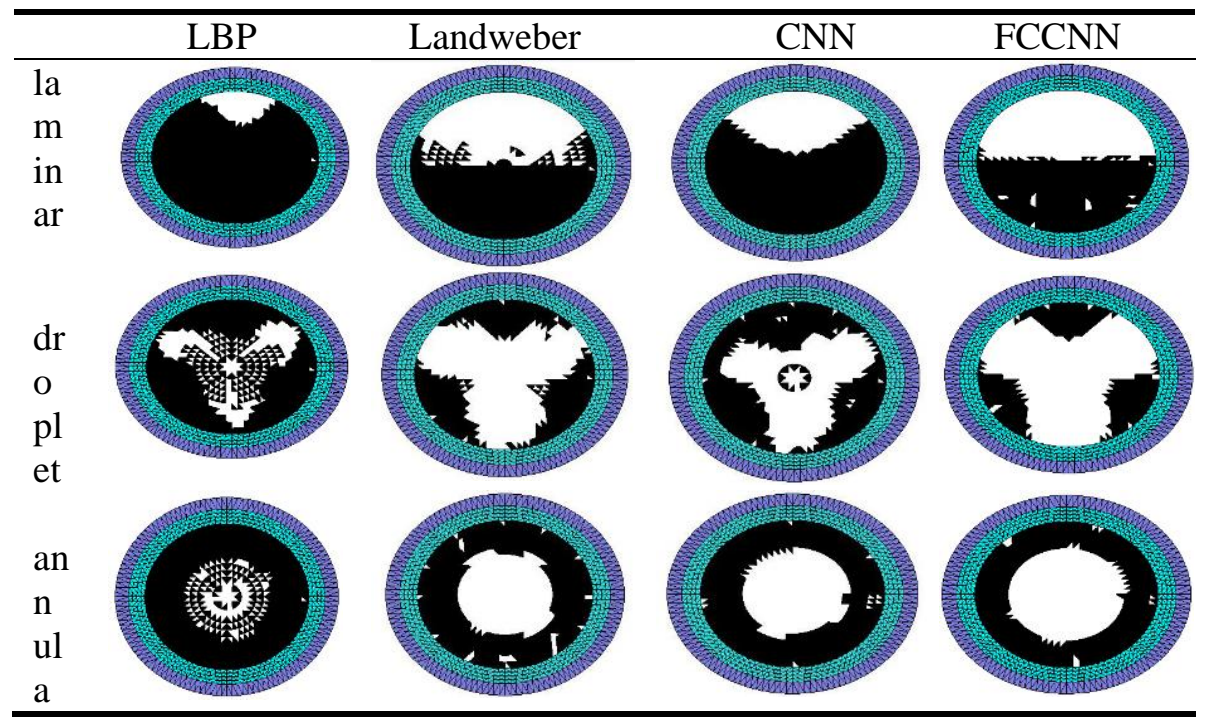

Figure 6. The Reconstruction Image of LBP Landweber CNN and FCCNN

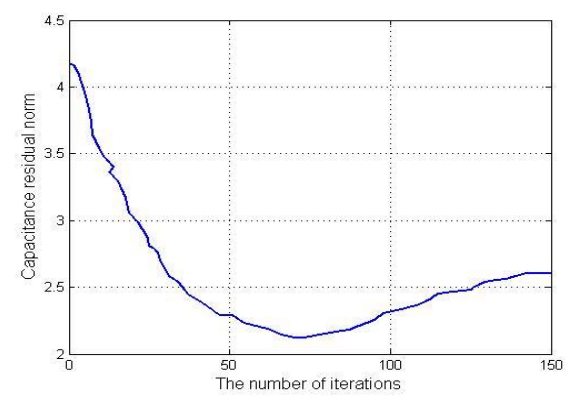

Figure 7. Iteration Number of Landweber

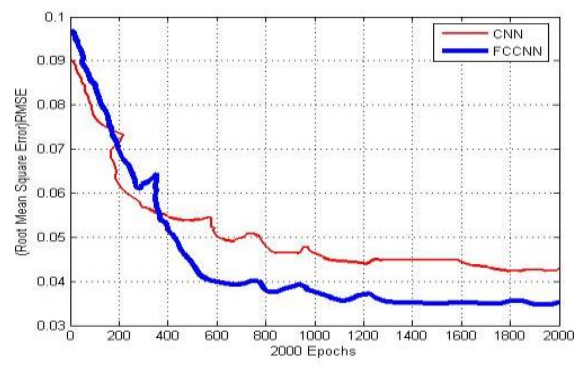

Figure 8. RMSE

Because the Landweber algorithm has the "semi convergence", we get the minimum of the capacitance residual after 75 iterations by experiments. So the image of the Landweber algorithm in Figure 7, is the effect of the 75 times iteration. In most cases, although it is the best image reconstruction algorithm in ECT system, but the slow convergence problem limits its application in the online real-time imaging field.

From the experimental results, we can see that the improved FCCNN algorithm is better than the traditional LBP algorithm and the iterative Landweber algorithm in image quality. In order to better verify the effectiveness of the improved algorithm and the image quality, we used the root mean square error (RMSE) to measure the difference between CNN, FCCNN and the original image (Figure 8). From this graph, we can see that the convergence speed of FCCNN algorithm is significantly faster than the CNN algorithm. In addition, the root mean square error of the algorithm is smaller, and the training results are more close to the original image. The image reconstruction times of four kinds of image reconstruction algorithm are shown in Table 2, which are obtained by Matlab simulation experiment. 


\section{Table 2. Image Reconstruction Time of Test Sample( ms)}

\begin{tabular}{ccccc}
\hline Algorithms & LBP & Landweber & CNN & FCCNN \\
\hline laminar & 0.68 & 137.32 & 1.98 & 1.12 \\
droplet & 0.72 & 153.57 & 2.02 & 1.34 \\
annular & 0.47 & 146.22 & 1.86 & 0.98 \\
\hline
\end{tabular}

As can be seen from Table 2, the imaging speed of LBP is obviously superior to other algorithms, but it can be seen from Figure 8, that the imaging quality is not high. Compared with the traditional Landweber algorithm, the image reconstruction time of CNN and FCCNN is significantly reduced, although their speed is less than LBP algorithm, and the image quality has been greatly improved. In addition, compared to the CNN algorithm, the imaging speed of improved FCCNN algorithm is also improved, which verifies the effectiveness of the improved algorithm.

\section{Conclusion}

This paper mainly discusses the system composition of the ECT system with 12 electrodes, positive and inverse problems theory and imaging principle. Moreover, image reconstruction algorithm for ECT system have also been further researched, and the ECT simulation system is established by using the Matlab software. The CNN and the improved FCCNN algorithm were applied to the image reconstruction of ECT system. Compared with the traditional Landweber and LBP algorithm imaging results, the effectiveness of the improved algorithm has been proved. Due to the training characteristics of CNN's multi hidden layer, with the increase of the depth of the model, if the training sample information is insufficient, the model will be over fitting. Therefore, the availability of sufficient sample is also an important factor affecting the imaging results. Due to $\mathrm{CNN}$ is mainly used to identify the rotation and translation of two-dimensional graphics. So for the stable flow pattern the identification imaging is more efficient. However, when the difference of the dielectric constant is less and flow pattern is complex, imaging is slightly inadequate, and the algorithm still needs further optimization and improvement.

\section{Reference}

[1] P. Zhenrui, Y. Hong and D. Haitang, "Electrical capacitance tomography technology research: a review", Sensor and micro system, vol. 28, (2009) September, pp. 6.

[2] T. Haijun and Z. Yunlong, "Research progress of electrical capacitance tomography", Chemical automation and instrumentation, (2012) Aug., pp. 8-9.

[3] G. Yanli, Z. Y. Gao and S. Fuqun, "The development and research of image reconstruction algorithm in electrical capacitance tomography", Sensors and micro systems, (2007) August, pp. 32-35.

[4] C. Deyun and L. Haoyang, "Analysis and comparison of image reconstruction algorithms for electrical capacitance tomography system", $\mathrm{Ph}$. D. Thesis, Harbin University of Science and Technology, (2006), pp. 6-8.

[5] Z. Yulei, G. Baolong and Y. Yun, "Research progress and analysis of electrical capacitance tomography technology", Journal of instrumentation and instrumentation, (2012) August, pp. 6-7.

[6] C. Deyun, W. Lili and C. Yu, "An algorithm for the reconstruction of electrical capacitance tomography based on improved trust region", Journal of instrumentation, vol. 6, (2010), pp. 57-60.

[7] Y. Jian, "Image reconstruction algorithm for electrical capacitance tomography", Northeastern University, (2012), pp. 62-64.

[8] H. Zhang and G. Rong, "An image reconstruction algorithm for electrical capacitance tomography[J]. Instrument and meter, vol. 3, (2005) August, pp. 6-7.

[9] L. Yandong, H. Q. Li and H. Zaigang, "Based on the rough neural network and feature extraction of ECT flow regime identification", Journal of Liaoning University, (2014) April.

[10] C. Deyun and C. Yu, "Inverse problem solving and image reconstruction algorithm for electrical capacitance tomography", Harbin University of Science and Technology, (2010), pp. 73-74.

[11] C. Linlin, "an image reconstruction algorithm for electrical capacitance tomography system", Micro computer information, (2007) August, pp. 72-78. 
[12] C. Jianyong, C. Juan and L. Xiaodong, "Preliminary study on hand gesture recognition based on convolutional neural network", Computer system application, vol. 5, (2015) April, pp. 68-74.

[13] C. Deyun and L. Mouzun, "Numerical analysis and parameter optimization of electric field characteristics of oil water two phase flow", Journal of system simulation, (2007) April, pp. 32-34.

[14] C. Deyun and W. Feihu, "Mathematical characteristics and image reconstruction algorithm for the inverse problem of electrical capacitance tomography", Harbin University of Science and Technology, (2014), pp. 14-15.

[15] Z. Xueming and W. Huaxiang, "Image reconstruction algorithm for electrical capacitance tomography", Tianjin University, (2004), pp. 23-25.

[16] C. Deyun, "Two phase flow electrical capacitance tomography research", Ph. D thesis, Harbin University of Science and Technology, (2006), pp. 24-26.

[17] K. K. Stone, "Convolutional neural network approach for buried target recognition in FL-LWIR imagery", Proceedings of SPIE J.M., (2014) May, pp. 112-115.

[18] H. Liang and C. Xianchang, "Research on the application and research of deep learning algorithm based on convolutional neural network", Zhejiang Gongshang University, (2014), pp. 36-37. 
International Journal of Signal Processing, Image Processing and Pattern Recognition Vol. 9, No. 11, (2016) 